\title{
Chip-Less and Battery-Less Subharmonic Tags for Wireless Sensing with Parametrically Enhanced Sensitivities and Dynamic Ranges
}

Hussein Hussein ( $\nabla$ h.hussein@northeastern.edu )

Northeastern University https://orcid.org/0000-0001-8772-9141

Matteo Rinaldi

Northeastern University

Marvin Onabajo

Northeastern University

Cristian Cassella

Northeastern University

\section{Article}

Keywords: wireless sensor nodes (WSNs), Internet-of-Things (IoT), Ultra-High-Frequency, Chip-Less and Battery-Less Subharmonic Tags

Posted Date: November 30th, 2020

DOl: https://doi.org/10.21203/rs.3.rs-81763/v1

License: (c) (i) This work is licensed under a Creative Commons Attribution 4.0 International License. Read Full License 


\title{
Chip-Less and Battery-Less Subharmonic Tags for Wireless Sensing with Parametrically Enhanced Sensitivities and Dynamic Ranges
}

\author{
Hussein M. E. Hussein ${ }^{1}$, Matteo Rinaldi ${ }^{1}$, Marvin Onabajo ${ }^{1}$, and Cristian Cassella ${ }^{1}$ \\ ${ }^{1}$ Northeastern University, Electrical and Computer Engineering Department, Boston, United States \\ *e-mail: h.hussein@northeastern.edu
}

\begin{abstract}
Massive deployments of wireless sensor nodes (WSNs) that continuously detect physical, biological or chemical parameters are needed to truly benefit from the unprecedented possibilities opened by the Internet-of-Things (loT). Just recently, new sensors with higher sensitivities have been demonstrated by leveraging advanced on-chip designs and microfabrication processes. Yet, WSNs using such sensors require energy to transmit the sensed information. Consequently, they either contain batteries that need to be periodically replaced or energy harvesting circuits whose low efficiencies prevent a frequent and continuous sensing and impact the maximum range of communication. Here, we report a new chip-less and battery-less tag-based WSN that fundamentally breaks any previous paradigm. This WSN, formed by off-the-shelf lumped components on a printed substrate, can sense and transmit information without any need of supplied or harvested DC power, while enabling full-duplex transceiver designs for interrogating nodes rendering them immune to their own self-interference. Also, even though the reported WSN does not require any advanced and expensive manufacturing, its unique parametric dynamical behavior enables extraordinary sensitivities and dynamic ranges that can even surpass those achieved by on-chip sensors. The operation and performance of the first implementation of this new WSN are reported. This device operates in the Ultra-High-Frequency range and is capable to passively and continuously detect temperature changes remotely from an interrogating node.
\end{abstract}

In the last decades, the continuously expanding Internet-of-Things (IoT) has created a plethora of new exciting possibilities within recently developed smart applications for structural health monitoring ${ }^{1}$, environmental surveys ${ }^{2}$, smart logistics ${ }^{3}$ and more. Nevertheless, such possibilities could be fully exploited only if low-cost and higher sensitivity wireless sensor nodes (WSNs) able to operate uninterruptedly were available for a massive-scale deployment ${ }^{4-7}$. For instance, the ability to strategically distribute thousands of such desired WSNs would aid to promptly detect and localize any behavioral anomalies in the structures of buildings, bridges and more, hence permitting to monitor their structural integrity ${ }^{8}$. Similarly, the same ability would allow to timely identify the occurrence of a fire ${ }^{9-12}$ in both indoor and outdoor settings, thus improving the safety of individuals and greatly reducing the losses in agriculture and in national resources that are more and more often experienced today. Also, according to recent studies, more than $20 \%$ of the food produced every year in the sole United States is wasted due to items in cold manufacturing and delivery chains ${ }^{13}$ exposed to not suitable temperatures, causing a financial loss of more than 200 billion dollars. The inadequate refrigeration of perishable food is also responsible for serious foodborne illnesses that annually cause more than one hundred thousand hospitalizations and thousands of deaths just in the $\mathrm{US}^{13}$. By enabling low-cost WSNs with long enough lifetime to continuously monitor all the processes in cold-chains and to promptly and reliably identify any specific items exposed to inadequate temperature, it would be possible to significantly lower these dramatic numbers, hence mitigating their serious consequences.

Any existing $\mathrm{WSN}^{14,15}$ used for remote sensing applications can be seen as the combination of a sensing system and a radio frequency (RF) front-end responsible to transmit and receive electromagnetic signals. The sensing system relies on a sensor to detect the variations of a specific parameter-of-interest $(\mathrm{PoI})$ with a sensitivity that strongly depends on the adopted sensing technology. In particular, the development of advanced manufacturing processes has recently enabled sensitive on-chip micro- and nano-electromechanical
(MEM/NEM) physical $^{12,16}$ and chemical ${ }^{17,18}$ sensors, consuming near-zero stand-by powers. Yet, the majority of the existing WSNs, including those using such miniaturized new sensors, still require considerable amounts of energy to transmit the sensed information to any other interrogating nodes or readers within the same network. As a result, they must rely on on-board batteries or, alternatively, on integrated harvesting circuits ${ }^{19}$, scavenging energy from the environment and use it to temporally sustain the transmission capabilities. Thanks to the recent advancement in zero-power sensor technologies $^{12}$, battery-powered WSNs can nowadays achieve extremely long lifetimes (nearly 10 years, limited by the self-discharge of their batteries) when deployed to detect time-critical but relatively rare events (i.e. operating predominantly in off- but alert-mode). Nevertheless, such lifetimes can be abruptly reduced to just few months when, instead, WSNs need to sense and transmit information many times per hour, thus demanding orders of magnitude higher average power levels than what consumed during their stand-by operational mode. In such more elaborate operational scenario, frequent periodical battery replacements are required, hence leading to high maintenance costs that can even be unsustainable when WSNs are deployed at hardly reachable locations or in harsh environments. Also, the increase of the number of deployed battery-powered WSNs is generating a fast growing environmental concern regarding the disposal of batteries in landfills. Similarly, any WSNs relying on on-chip harvesting circuits are also hardly usable when a frequent detection of any PoIs is required. In fact, both the maximum communication range and the highest detection rate achievable through these WSNs are severely affected by the inability of the currently available rectifying circuits to exhibit acceptable efficiencies when receiving RF power levels significantly lower than $1 \mathrm{~mW}^{20}$. So, in order to enable WSNs that can be frequently interrogated without relying on any batteries or harvesting circuits, a growing attention has been recently paid to chip-less and battery-less tag-based WSNs. These WSNs are printable on easily disposable substrates, thus enabling exceptionally low manufacturing costs ${ }^{7}$, while being equipped with sensing capabili- 

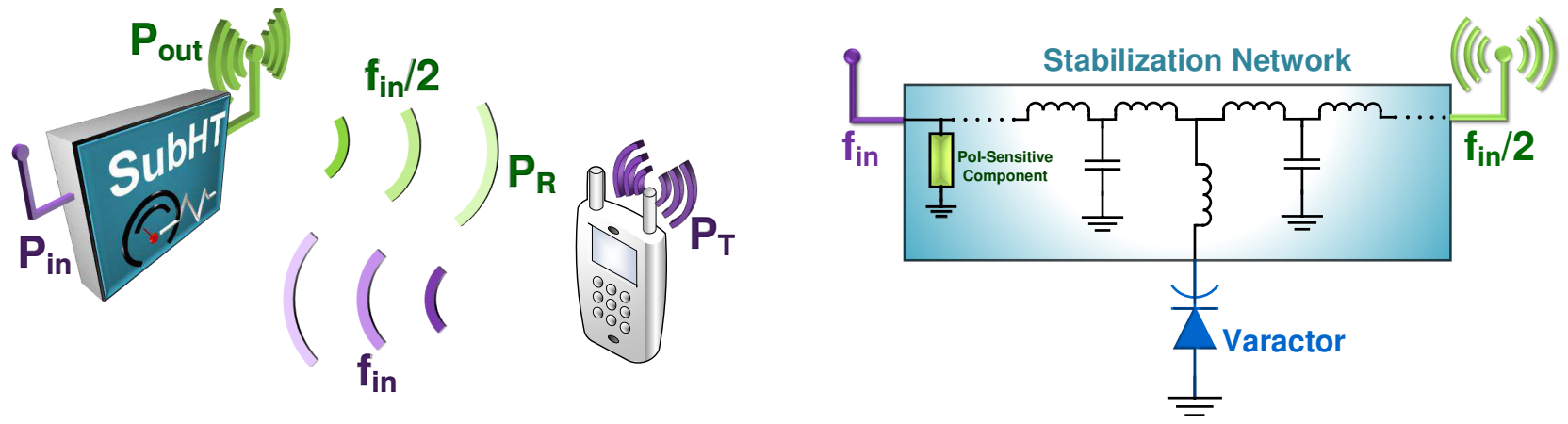

d
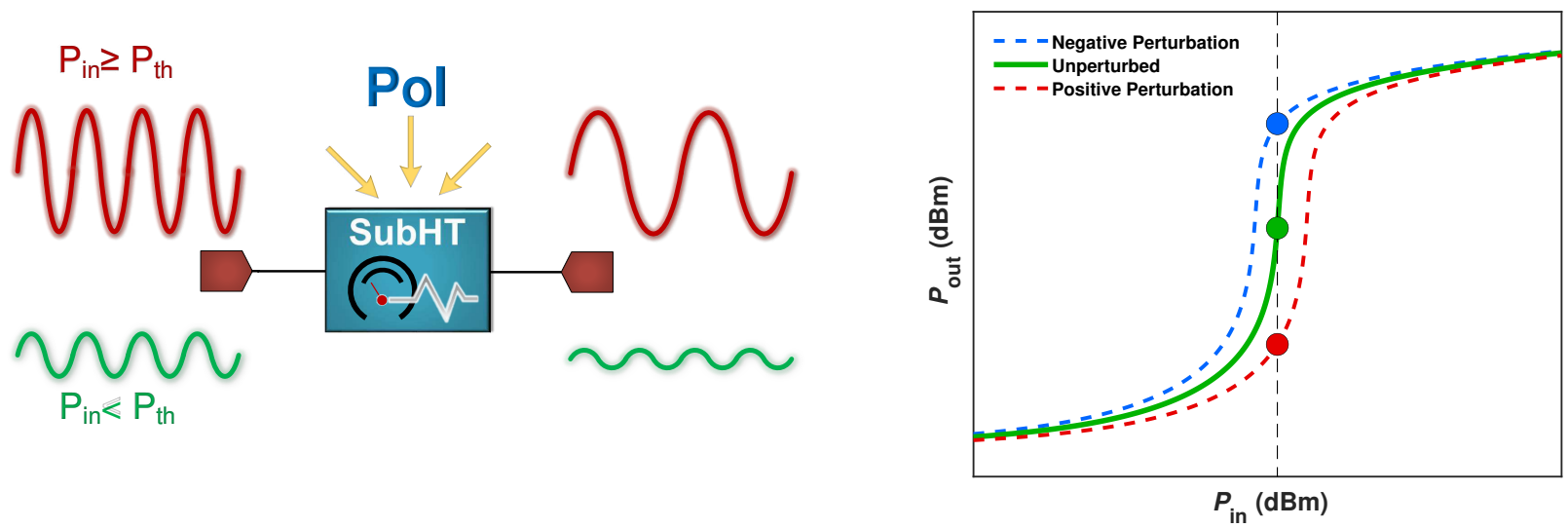

Fig. 1 I A Sub-Harmonic Tag (SubHT) and its unique operational features. a, Schematic of an envisioned SubHT-enabled wireless sensing architecture. It allows to passively and remotely sense any targeted PoIs. Also, the sensed information is radiated back from the SubHT towards the interrogating node by using a passively generated carrier frequency $\left(f_{\text {in }} / 2\right)$ that is half of the interrogating frequency $\left(f_{\text {in }}\right)$. b. Circuit schematic representation of a generic SubHT. This includes a varactor and a passive network of off-the-shelf lumped components acting as a stabilization network for the large-signal periodic regimes driven by the SubHT received input power $\left(P_{i n}\right)$. Also, this network embodies one component that is sensitive to the specific PoI and that is responsible for the activation of the unique dynamics leveraged by the SubHT to sense the PoI. c, A graphic representation of the typical input and output signals characteristics of a SubHT, for input power levels $\left(P_{i n}\right)$ lower (in green) or higher (in red) than the SubHT parametric power threshold $\left(P_{t h}\right)$. d, Typical output power $\left(P_{\text {out }}\right) v s . P_{i n}$ characteristic of a SubHT when not perturbed (in green) by the $P o I$ or, alternatively, when subject to a positive (in red) or negative (in blue) variations of the PoI.

ties. Yet, in order to achieve a small size and a long communication range, while rendering any interrogating nodes able to separate the transmitted and received data streams, the existing chip-less and battery-less tag-based WSNs must rely on advanced resonant components with exceptionally high quality factors $(Q)$, like surface acoustic wave (SAW) ${ }^{21}$ devices. Although the use of such high- $Q$ components comes with significantly higher manufacturing costs ${ }^{7}$, these devices are key, when used in conventional tag-based WSNs, to ensure that any interrogating nodes can distinguish the received sensed information from their self-interference and from any occurring environmental electromagnetic echos of their output signals. Just recently, in order to avoid using any expensive high- $Q$ components, a class of chip-less and battery-less tag-based WSNs known as harmonic tags (HTs) has been proposed ${ }^{22,23}$. These WSNs rely on unbiased nonlinear devices, such as varactors or Shottkey diodes, to deliver the sensed information through output signals that have twice the frequency of the interrogating ones, thus being easily distinguishable, once received by the interrogating nodes, from any undesired signals with the same frequency used by the interrogating one. Yet, the output signals of harmonic tags show power levels that are lower than those of their input signals by a large amount known as conversion loss (CL) that, depending on the technology of the nonlinear variable capacitor, can even exceed $35 \mathrm{~dB}^{23}$ when the received input power levels are lower than $-15 \mathrm{dBm}$. In addition, since the sensed information is transmitted at twice the frequency of the interrogating signals, harmonic tags inherently suffer from a $6 \mathrm{~dB}$ higher path-loss than traditional single-frequency counterparts, thus further reducing the signal-to-noise ratio (SNR) at the receiver of their interrogating nodes and, consequently, the maximum communication range.

In this Article, we present the first prototype of a novel class of chip-less and battery-less tag-based WSNs, referred to as subharmonic tags (SubHTs) (Fig. 1a). SubHTs break any previous paradigm related to the design of chip-less and battery-less tag-based WSNs by making it possible to remotely and continuously sense PoIs with extraordinary sensitivities and dynamic ranges, yet relying on low-cost off-the-shelf lumped components assembled on printed 
substrates. Also, similarly to any previously reported harmonic tags, SubHTs transmit the sensed information over a dedicated channel, distant from the one leveraged to interrogate them. However, differently from any previous counterparts, this full-duplex characteristic is achieved by strategically operating in regions where a parametrically originated period-doubling mechanism is active. This mechanism allows SubHTs to transmit the sensed information through a dedicated passively generated carrier frequency $\left(f_{\text {out }}\right)$, which is half of the one used by the interrogating signal $\left(f_{\text {in }}=2 f_{\text {out }}\right)$. Regardless of the very low input power levels $(<-18 \mathrm{dBm})$ at which SubHTs can operate and despite the fact that no DC biasing voltage is used, SubHTs generate the output signal from the received input power more efficiently than harmonic tags, thus enabling significantly lower CLvalues. Furthermore, since for any chosen $f_{\text {in }}$ value $f_{\text {out }}$ is always one fourth of the output frequency that would be used if harmonic tags were adopted, SubHTs inherently enable a $12 \mathrm{~dB}$ reduction in the path-loss affecting the portion of their output signal reaching the interrogating nodes. These unique features enable orders of magnitude higher SNRs at the receiver of the interrogating nodes than what has ever been possible to achieve through harmonic tags, hence paving the way towards a more accurate wireless sensing and a longer communication range. Furthermore, we show that the unexplored parametric dynamics leveraged by SubHTs ${ }^{24-27}$ also allow to massively boost the sensitivity and the dynamic range attained by offthe-shelf commercial sensors, thus providing the means to achieve superior sensing capabilities without requiring advanced on-chip sensors like the recently developed MEM/NEM components.

In order to demonstrate the unique characteristics exhibited by SubHTs, the operation and performance of the first SubHT prototype made of off-the-shelf lumped components are described here. This device operates at $f_{\text {in }}$ equal to $886 \mathrm{MHz}$ and remotely measures temperature at 4 meters from a complementary interrogating node. Despite the fact that this SubHT is not relying on any advanced components with high temperature-sensitivity and high dynamic range but only on a commercial off-the-shelf thermistor, it can show a sensitivity $\left(S_{\max }\right)$ and a dynamic range that are orders of magnitude higher than what is achievable when the same thermistor is used as a separate sensor. In the next section, we will discuss the general principle of operation of SubHTs. Afterwards, we will focus on the main design and performance characteristics of the built SubHT prototype.

\section{Principle of operation}

Independently of the targeted sensing parameter, any SubHT can be described as a two-port electrical network formed by an un-biased variable capacitor and a set of lumped electrical passive elements (Fig. 1b). This set includes a component, such as a separate commercial off-the-shelf sensor, with an electrical impedance dependent on the specific PoI. The two ports of any SubHTs are connected to properly sized antennas, enabling the simultaneous reception and transmission of signals from and to the interrogating nodes. The technology (planar, wire, aperture, etc.) and design characteristics of such antennas can be chosen based on the targeted application and other system level requirements. Depending on the strength and on the frequency of its input signal, a SubHT can exhibit operational regions where it undergoes a period-doubling mechanism ${ }^{26,28}$. In such regions, it relies on the energy coming from the interrogating node, at a frequency $f_{\text {in }}$, to passively generate a strong output signal at $f_{\text {in }} / 2$ (i.e. $f_{\text {out }}$ ), which is radiated back to the interrogating node. The activation of such period-doubling mechanism (Fig. 1c) occurs through a super-critical bifurcation ${ }^{28}$ triggered by the power $\left(P_{i n}\right)$ of the SubHT input signal. In particular, for $P_{i n}$ values exceeding a certain threshold (known as parametric threshold, $P_{t h}$ ), SubHTs exhibit a steep but continuous $P_{\text {out }}$ vs. $P_{\text {in }}$ characteristic (Fig. 1d), where $P_{\text {out }}$ is the output power at $f_{\text {out }}$ delivered to the antenna used for transmission. $P_{t h}$, which designates the minimum input power at which a SubHT can operate, is set by the junction capacitance and tuning range exhibited by the adopted varactor, along with the impedances that such variable capacitor sees at both $f_{\text {in }}$ and $f_{\text {out }}{ }^{27}$. These impedances are set by the equivalent passive network formed by the selected lumped components excluding the varactor. Such network acts as a stabilization network for the non-autonomous periodic regimes generated by the interrogating signal through the large modulation of the varactor's capacitance. The SubHT lumped components are selected to minimize $P_{t h}$ given a desired $f_{\text {in }}$ value. Yet, by including the chosen component with impedance dependent on the targeted PoI within the stabilization network, any change in the strength exerted on the SubHT by such PoI results into a corresponding change of $P_{t h}$, hence activating a previously unexplored dynamical behavior that is leveraged here for the first time (Fig. 1d). Due to the steep slope of the $P_{\text {out }} v s . P_{\text {in }}$ characteristic for $P_{i n}$ approaching $P_{t h}$ and due to the fact that the power at $f_{\text {out }}$ is only generated for $P_{i n}$ higher than $P_{t h}$, any induced variations of $P_{t h}$, even if small, produces an extremely large change of $P_{\text {out }}$ and, consequently, of the power received $\left(P_{R}\right)$ at $f_{\text {out }}$ by the interrogating device. Such change can span over several orders of magnitude, even when only small perturbations to the SubHT operational point are caused by the PoI. This unique dynamical feature provides the means to achieve a sensitivity to the $P O I$ and a dynamic range that greatly exceed what is possible when the selected SubHT component with electrical response dependent on the $P o I$ is used as an independent sensor. In other words, SubHTs pave the way towards tag-based WSNs that can surpass, electronically, the limited sensitivity of their sensitive element, instead of requiring more advanced technologies that demand higher fabrication complexities or special operating conditions unsuitable for a massive-scale deployment. By analyzing the received power $\left(P_{R}\right)$ at $f_{\text {out }}$, the interrogating node can then remotely assess the strength of the PoI at the SubHT location. Thanks to the fact that SubHTs can couple the sensed information to a different carrier frequency from the one used to interrogate them, they don't need high quality factor components. Instead, SubHTs enable full-duplex transceiver architectures for the interrogating nodes, simply relying on two filtering components, centered at $f_{\text {in }}$ and $f_{\text {out }}$, to separate simultaneously transmitted and received data streams.

Furthermore, it is also crucial to point out that due to the SubHTs unique dynamics, the generation of their sub-harmonic output signal from $P_{\text {in }}$ can be significantly more efficient than the corresponding production of a high-order harmonic in any harmonic tags. Such unexplored feature is enabled by the capability of any parametric systems operating above threshold to more efficiently transform the energy stored by their nonlinear reactances at the driving frequency into power at the desired sub-harmonic output frequency. This can be verified, for instance, by monitoring the different trends of the power dependent quality factor $\left(Q_{v}\right.$, Supplementary Fig. 4.) exhibited by an ideal lossless nonlinear reactance, connected either to a lossless stabilization network to enable a frequency division with minimum $P_{t h}$ or to a circuit exploiting the same topology used for the stabilization network, yet engineered to allow a frequency doubling functionality with minimum CL. These frequency dividing and frequency doubling systems operate with the same input frequency $\left(f_{c}\right)$ and input power $\left(P_{c}\right)$ but with an output frequency being either half or twice $f_{c}$ (Fig. 2). As we rely on these two exemplificative systems to assess the capability of the same nonlinear reactance to generate different 


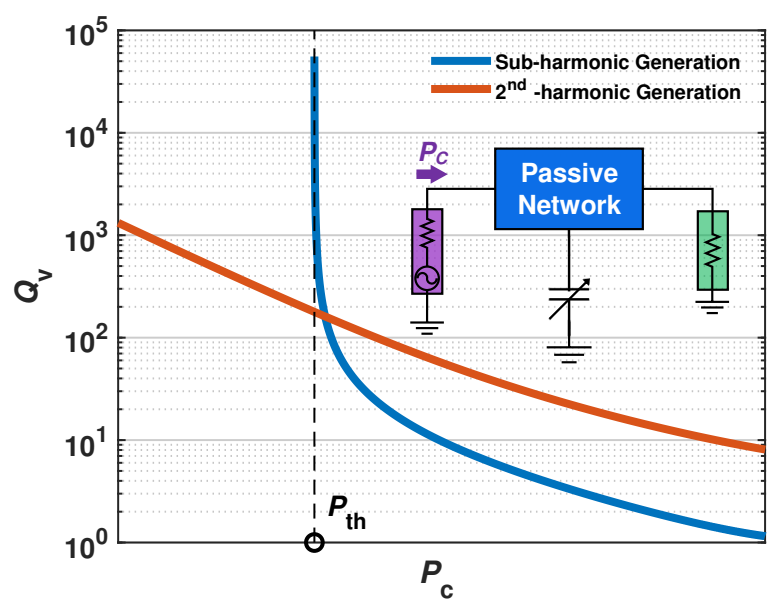

Fig. 2 | The power dependent quality factor exhibited by an ideal largely modulated reactance used for frequency division or frequency doubling Typical trends of $Q_{v}$ vs. $P_{c}$ attained through circuit simulations and relative to an ideal nonlinear reactance, independently used by two same-order and same-topology passive circuits respectively optimized for frequency division by two (in blue) or for frequency doubling (in red). More details about the simulation strategy we followed to extract these trends are reported in Supplementary Fig. 4.

desired output frequencies, $Q_{v}$ is of great interest as it maps the ratio between the imaginary part of the modulated reactance impedance at $f_{c}$ and its nonlinearly generated resistance $\left(R_{\text {conv }}\right)$. This resistance captures the effects of the capacitance modulation at $f_{c}$ on the energy transformation between input and output frequencies, thus progressively increasing as higher $P_{c}$ values are used. Also, differently from $\mathrm{CL}, Q_{v}$ is independent of the matching characteristics relative to the circuits ports, hence being a more adequate parameter to assess the intrinsic conversion capabilities granted by the same nonlinear reactance when used in the two analyzed systems. In particular, while $Q_{v}$ diverges for $P_{c}$ tending to zero (for the frequency doubling circuit) or to $P_{t h}$ (for the parametric frequency dividing circuit), due to the decreasing capacitance modulation lowering $R_{c o n v}$, it progressively reduces as $P_{c}$ is increased. In particular, by comparing the trends of $Q_{v}$ vs. $P_{c}$ relative to the two investigated circuits, a significantly lower $Q_{v}$ value can be found, for $P_{c}$ higher than $P_{t h}$, when the nonlinear reactance is used to parametrically generate a sub-harmonic output signal, like in SubHTs, rather than create a second harmonic one, like in any previously reported harmonic tags (Fig. 2). As a result, for low $P_{c}$ values, the CL value achieved by SubHTs can be smaller than the corresponding value in harmonic tags, thus allowing to increase the SNR at the receiver of the interrogating nodes without requiring more power to be transmitted by the same nodes. Moreover, it is important to point out that since $P_{\text {out }}$ has a frequency that is one fourth of the output one adopted for the same driving frequency by harmonic tags, the SNR improvement enabled by SubHTs is further amplified (by $12 \mathrm{~dB}$ in free-space) due to a reduction in the path-loss affecting $P_{\text {out }}$ before reaching any interrogating nodes.

\section{An Ultra-High-Frequency (UHF) SubHT for temperature sensing}

In order to experimentally demonstrate the unique performance features of SubHTs, we decided to build a SubHT prototype targeting a remote and continuous temperature $(T)$ sensing. This prototype was designed and assembled on a printed circuit board (PCB) made

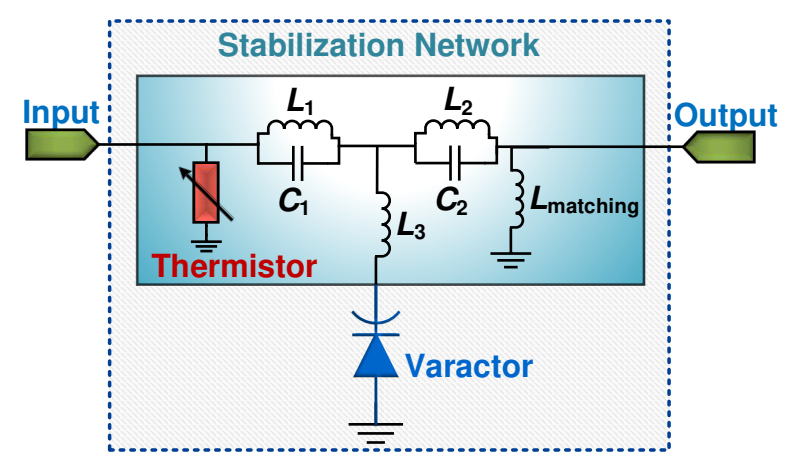

Fig. 3 | Circuit schematic of the realized SubHT for temperature sensing. The components forming the stabilization network of the built SubHT are shown, including the off-the-shelf thermistor used to activate the unique temperature-sensitive dynamics leveraged during the sensing operation. The values and model-numbers of all components in the circuit and a picture of the fabricated SubHT are available in the Supplementary Material (Supplementary Fig. 1 and Supplementary Table 1).

of FR-4, relying on off-the-shelf lumped components including two capacitors $\left(C_{1}\right.$ and $\left.C_{2}\right)$, four inductors $\left(L_{1}, L_{2}, L_{3}, L_{\text {matching }}\right)$, one varactor and a commercial thermistor (Fig. 3). The thermistor was used as the required sensitive element in the SubHT stabilization network, allowing its unique temperature-sensitive dynamics. Following our recent theoretical investigation on the stability of varactor-based parametric systems ${ }^{27}$, and given the impedance exhibited at room temperature by the selected thermistor, the inductors and capacitors of the built SubHT were selected to minimize $P_{t h}$ at $f_{\text {in }}$ equal to $886 \mathrm{MHz}$. This was done by satisfying four resonant conditions allowing the maximum voltage level across the varactor at $f_{\text {in }}$, the minimum leakage of $P_{\text {out }}$ towards the receiving antenna and the lowest impedance magnitude seen by the varactor at $f_{\text {out }}$. In other words, such design conditions simultaneously enable the largest modulation depth of the varactor's capacitance, the highest output power, and the lowest loss that is to be parametrically compensated in order to trigger the desired sub-harmonic oscillation in the circuit. We characterized the unique temperature sensing capabilities of the SubHT by placing it on a digitally controlled hotplate to vary the $T$ value at the SubHT location from $25^{\circ} \mathrm{C}$ to $60^{\circ} \mathrm{C}$ with a step of $2.5^{\circ} \mathrm{C}$. The SubHT input and output ports were connected to two synchronized network analyzers, respectively acting as a $50 \Omega$ signal generator at $f_{\text {in }}$ and as a $50 \Omega$ power meter at $f_{\text {out }}$. The measured $P_{\text {out }} v s . P_{\text {in }}$ characteristics for all the explored $T$ values are reported (Fig. 4a), along with the closely matching corresponding ones we found through circuit simulations (Fig. 4b). As expected, a super-critical bifurcation was found for all the explored $T$ values, marking the transition between the SubHT operational regions without frequency division and the ones with frequency division. In particular, $P_{t h}$ values as low as $-18.5 \mathrm{dBm}$ were measured along with $C L$ values approaching $21 \mathrm{~dB}$, which are significantly lower than the ones of any reported harmonic tags relying on unbiased nonlinear reactances ${ }^{23}$ to avoid using batteries or energy harvesters. Furthermore, as the temperature at the SubHT location was varied, we noticed a clear monotonic increase of $P_{t h}$ caused by a temperature-driven change of the impedance seen by the varactor at $f_{\text {in }}$. Due to the steep slope of the $P_{\text {out }} v s . P_{\text {in }}$ characteristic exhibited in proximity of the super-critical bifurcation, such a shift in $P_{t h}$ can produce a large variation of $P_{\text {out }}$ that provides the means to achieve the superior sensing capabilities reported in this work. In fact, by strategically selecting a $P_{i n}$ value close to the specific 

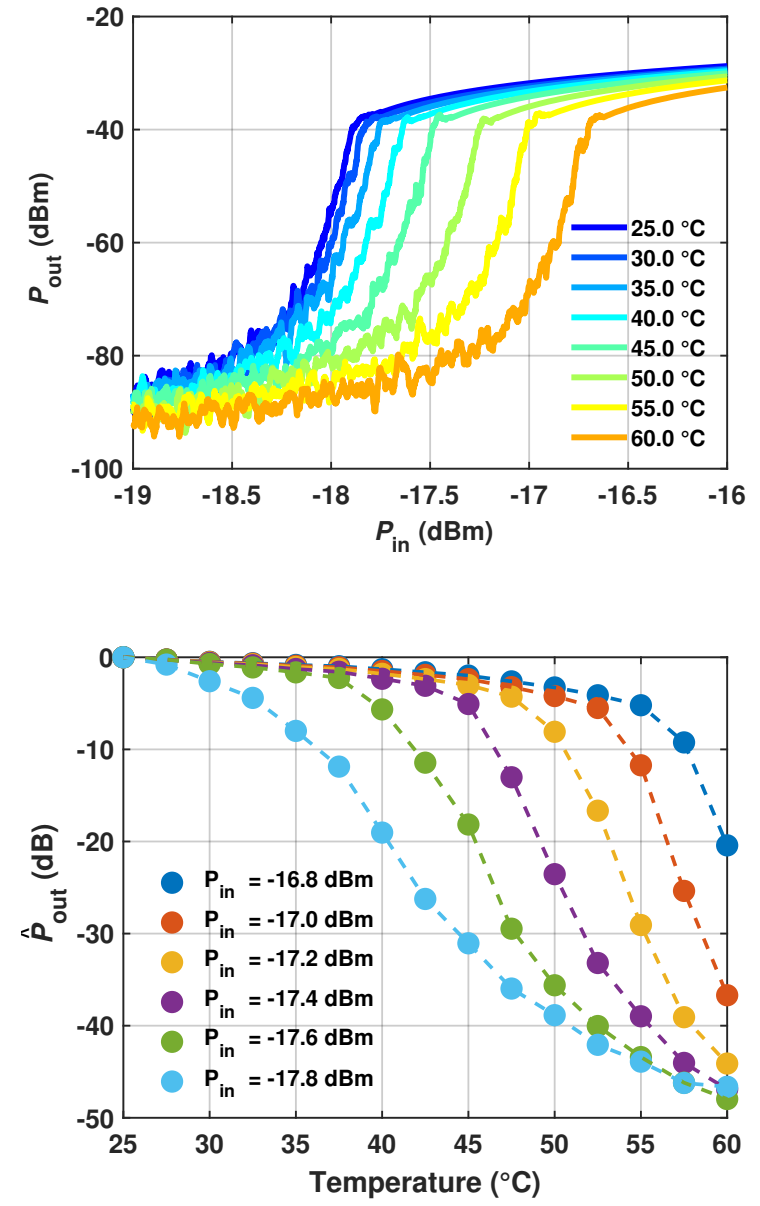

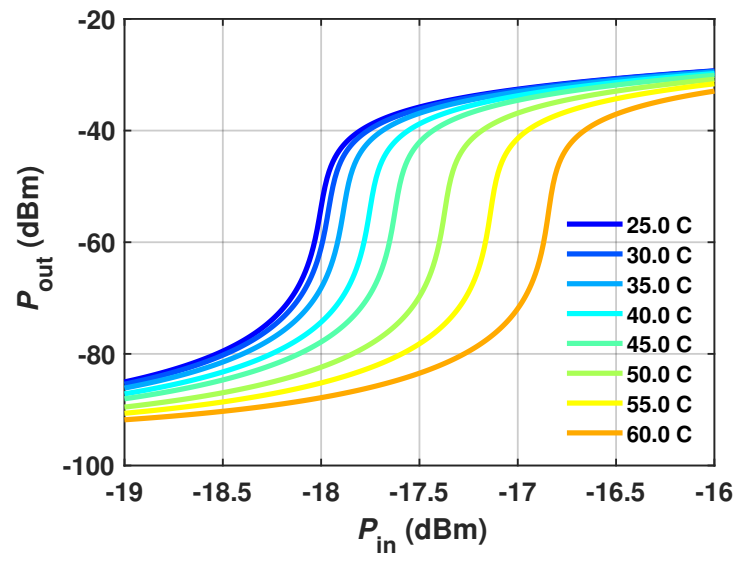

d

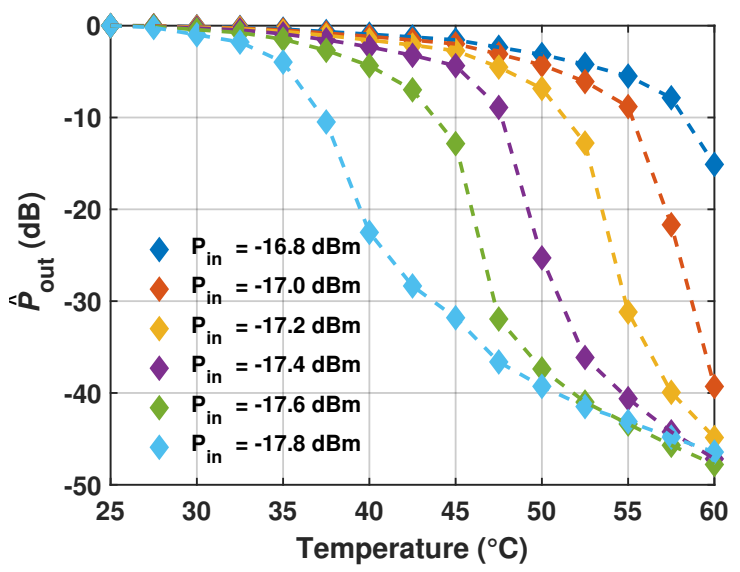

Fig. 4 | Evaluation of the sensing capabilities of the fabricated SubHT. a,b, Measured (a) and simulated (b) $P_{\text {out }} v s . P_{\text {in }}$ trends of the fabricated SubHT, at $f_{\text {in }}=886 \mathrm{MHz}$ and for different temperatures $(T \mathrm{~s})$ ranging from $25^{\circ} \mathrm{C}$ to $60^{\circ} \mathrm{C}$. c,d, Measured (c) and simulated (d) $P_{\text {out }} v s . T$ trends of the fabricated SubHT for different $P_{i n}$ values close to the $P_{t h}$ value extracted at $25^{\circ} \mathrm{C}$. All the reported curves $(\mathbf{a}, \mathbf{b}, \mathbf{c}, \mathbf{d})$ were extracted through a wired characterization experiment. More details about the experimental set-up and the followed simulation approach are discussed in the Supplementary Material (Supplementary Fig. 2).

$P_{t h}$ value measured at $25^{\circ} \mathrm{C}$, this SubHT can obtain extraordinary sensitivities and dynamic ranges that cannot be reached otherwise. This was confirmed, through both direct measurements and circuit simulations, by extracting the corresponding $P_{\text {out }}$ values for different $P_{\text {in }}$ close to $-18.5 \mathrm{dBm}$ and when considering the same analyzed $T$ values. The extracted values $\left(\hat{P}_{\text {out }}\right)$ from both our measurements and simulations, normalized to the corresponding $P_{\text {out }}$ values at $25^{\circ} \mathrm{C}$, are shown in Fig. 4c and Fig. 4d respectively. As evident, the built SubHT can exhibit remarkable ratios $\left(\Delta \hat{P}_{\text {out }}\right)$ between the $\hat{P}_{\text {out }}$ values extracted at $25^{\circ} \mathrm{C}$ and $60^{\circ} \mathrm{C}$. This allows to reach average temperature sensitivities $\left(S_{\text {avg }}=\Delta \hat{P}_{\text {out }} / \Delta T\right.$, being $\Delta T$ the size of the explored temperature range) as high as $1.4 \mathrm{~dB} /{ }^{\circ} \mathrm{C}$. Such $S_{\text {avg }}$ value is 20 times higher than what is attainable $\left(0.07 \mathrm{~dB} /{ }^{\circ} \mathrm{C}\right)$ when the thermistor included in the built SubHT is used as a separate sensor, altering the power flow between the two electrical ports of a dedicated optimized circuit exposed to the same temperature changes (Fig. 5). In addition, the SubHT shows a maximum value $\left(S_{\max }\right)$ for the temperature sensitivity across the investigated temperature range, defined as the magnitude of the largest slope of the $\hat{P}_{\text {out }} v s . T$ trend, of $6.2 \mathrm{~dB} /{ }^{\circ} \mathrm{C}$, measured at $P_{\text {in }}$ equal to $-17 \mathrm{dBm}$ and around a $T$ value of $57.5^{\circ} \mathrm{C}$. In particular, we found that, by operating at such optimal working condition, the built SubHT not only exhibits the highest sensitivity but also attains the lowest temperature resolution, equal to $0.002^{\circ} \mathrm{C}$ (see Supplementary Fig. 5 for a measured trend of resolution vs. $P_{\text {in }}$ at $57.5^{\circ} \mathrm{C}$ ). This proves that the predominant noise source limiting the value of the minimum detectable temperature change is not the adopted thermistor but the network analyzer used for the read-out. Furthermore, the SubHT shows a large dynamic range of $48 \mathrm{~dB}$. The measured $S_{\max }$ and dynamic range values are respectively 37 times and 35,000 times higher than the corresponding values $\left(0.17 \mathrm{~dB} /{ }^{\circ} \mathrm{C}\right.$ and $2.6 \mathrm{~dB}$ ) attained when the thermistor in the SubHT circuit is used as a separate temperature sensor (Fig. 5). Finally, the adjusted R-squared value relative to the SubHT measured $\hat{P}_{\text {out }} v s . T$ trend can reach 0.9669 , demonstrating a good linearity between temperature and $\hat{P}_{\text {out }}$. So, our measured results demonstrate that SubHTs can surpass the fundamental limits of the sensitive component in their stabilization networks. A comparison between $S_{\max }$ and the maximum sensitivity values attained by other recently reported intensity-level temperature sensors is provided in Table 1. As evident, the measured SubHT can exhibit a higher temperature sensitivity than any other previously reported counterparts, yet not requiring any active and large sensing set-ups, such as those needed when relying on optical 


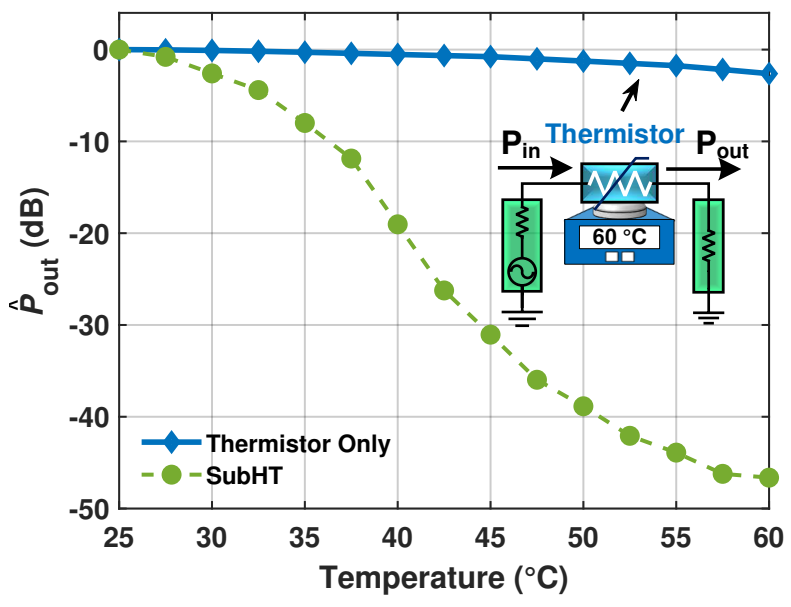

Fig. 5 I Surpassing the limits in the achievable sensitivity. A comparison of the $\hat{P}_{\text {out }}$ values attained by the built SubHT (in green), for the different investigated temperatures, with the corresponding $\hat{P}_{\text {out }}$ values (in blue) that would be attained, instead, if the thermistor used by the SubHT was individually utilized as the temperature sensor. For clarity, the circuit schematic used for the evaluation of the latter case is also displayed in the in-set. components and systems, or any advanced integrated complementary metal-oxide-semiconductor (CMOS) and SAW devices.

After characterizing its sensing capabilities through a wired setup, we designed a new experiment to demonstrate the ability of the built SubHT to operate as a fully passive WSN, remotely sensing any temperature variations even when operating in uncontrolled electromagnetic environments like the authors' laboratories at Northeastern

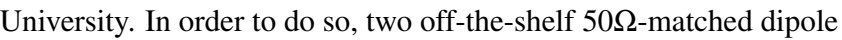
antennas were connected at the SubHT's input and output ports. This rendered the SubHT simultaneously able to receive its interrogating signal wirelessly and to radiate its parametrically generated output signal. Moreover, two additional antennas, identical to those used by the SubHT, were connected to the same network analyzers from the previous wired characterization (Fig. 4c). This allowed to emulate a complementary wireless interrogating transceiver like the one conceptualized in Fig. 1a, able to radiate an interrogating signal at $886 \mathrm{MHz}$ with power $P_{T}$ while simultaneously receiving a portion (i.e., $P_{R}$ ) of $P_{\text {out }}$ at $443 \mathrm{MHz}$. The two network analyzers were positioned 4 meters away from the SubHT and next to each other, as depicted in Fig. 6a. As in our former experiment, the SubHT was placed on top of a digitally controlled hotplate to set the temperature value at its now remote location. All the antennas were physically oriented to minimize any polarization losses that would lower the power received by the SubHT (i.e., $P_{i n}$ ) and reduce $P_{R}$. The adoption of an additional amplification stage, connected between the output port of the network analyzer used for transmission and the adjacent antenna, allowed to sweep $P_{T}$ between $20 \mathrm{dBm}$ and $40 \mathrm{dBm}$, while varying $T$ at the SubHT location as in our former wired experiment. The measured $P_{R} v s . P_{T}$ characteristic for the explored $T$ values is reported in Fig. 6b. As evident, distinguishable and monotonic temperature-driven changes of the $P_{T}$ values triggering the sub-harmonic oscillation $\left(P_{t h}^{T}\right)$ in the SubHT can be observed, even when operating the SubHT as a WSN. In particular, $P_{t h}^{T}$ values between $27 \mathrm{dBm}$ and $34 \mathrm{dBm}$ were found as $T$ was varied from $25^{\circ} \mathrm{C}$ to $60^{\circ} \mathrm{C}$. Such high power levels are needed to compensate for the losses encountered during the electromagnetic propagation and for
Table 1 I Comparison with other temperature sensors. The maximum temperature sensitivity and the corresponding resolution of the SubHT (i.e., $S_{\max }$ ) are compared with those recently demonstrated by other previously reported counterparts, over different temperature ranges and through other sensing technologies.

\begin{tabular}{|c|c|c|c|c|}
\hline $\begin{array}{c}\text { Sensor } \\
\text { Prototypes }\end{array}$ & $\begin{array}{c}\text { Sensing } \\
\text { Technology }\end{array}$ & $\begin{array}{c}\text { Temperature } \\
\text { Range } \\
\left({ }^{\circ} \mathrm{C}\right)\end{array}$ & $\begin{array}{c}\text { Max } \\
\text { Sensitivity } \\
\left(\mathrm{dB} /{ }^{\circ} \mathrm{C}\right)\end{array}$ & $\begin{array}{c}\text { Min } \\
\text { Resolution } \\
\left({ }^{\circ} \mathrm{C}\right)\end{array}$ \\
\hline This work & Parametric & $25-60$ & 6.2 & 0.002 \\
\hline Ref. $^{29}$ & Optical & $22-27$ & 0.058 & - \\
\hline Ref. $^{30}$ & Optical & $25-65$ & 0.42 & - \\
\hline Ref. $^{31}$ & Optical & $26-100$ & 0.23 & - \\
\hline Ref. $^{32}$ & Optical & $47-63$ & 2.26 & - \\
\hline Ref. $^{33}$ & Optical & $22-40$ & 2.1 & 0.0005 \\
\hline Ref. $^{34}$ & Optical & $40-100$ & 0.24 & - \\
\hline Ref. $^{35}$ & Optical & $15-60$ & 0.22 & - \\
\hline Ref. $^{36}$ & Optical & $20-75$ & 0.03 & 0.03 \\
\hline Ref. $^{37}$ & Optical & $30-80$ & 0.1 & 0.0098 \\
\hline Ref. $^{38}$ & Optical & $22-60$ & 0.13 & - \\
\hline Ref. $^{21}$ & SAW & $25-300$ & 0.16 & - \\
\hline Ref. $^{39}$ & SAW & $35-118$ & 0.13 & - \\
\hline Ref. $^{40}$ & SAW & $25-300$ & 0.065 & 0.15 \\
\hline Ref. $^{41}$ & SAW & $20-100$ & - & 0.016 \\
\hline Ref. $^{42}$ & CMOS & $0-100$ & - & 0.0582 \\
\hline Ref. $^{43}$ & CMOS & $30-49$ & 0.027 & 0.003 \\
\hline Ref. $^{44}$ & CMOS & $-20-60$ & - & 0.21 \\
\hline
\end{tabular}

those introduced by all the adopted electrical components and connections. Since for $P_{T}$ higher than $P_{t h}^{T}$ the measured $P_{R}$ values are $20 \mathrm{~dB}$ or less above the noise floor of the network analyzer used to extract them, the wireless sensing of $T$ can be achieved across the entire explored temperature range for $P_{T}$ values higher than $34 \mathrm{dBm}$. The measured and simulated $P_{R}$ values $\left(\hat{P}_{R}\right)$ for all the investigated $T$ values and normalized with respect to the corresponding $P_{R}$ values at $25^{\circ} \mathrm{C}$ are reported in Fig. $6 \mathrm{c}$ and Fig. $6 \mathrm{~d}$ respectively. As evident, a large difference $\left(\Delta \hat{P}_{R}\right)$ between the $P_{R}$ values at $25^{\circ} \mathrm{C}$ and $60^{\circ} \mathrm{C}$ was found for $P_{T}$ equal to $34 \mathrm{dBm}$, resulting in an average sensitivity $\left(S_{a v g}^{w}\right)$ of $0.6 \mathrm{~dB} /{ }^{\circ} \mathrm{C}$ and in a dynamic range of $21 \mathrm{~dB}$. A maximum measured sensitivity $\left(S_{\max }^{w}\right)$ of $3 \mathrm{~dB} /{ }^{\circ} \mathrm{C}$ was detected for the same $P_{T}$ value. It is worth pointing out that the measured $S_{a v g}^{w}$ and $S_{\max }^{w}$ values exceeds by nearly 4 and 19 times the corresponding ones just recently demonstrated by using advanced mm-wave imaging circuits that analyze the temperature sensitive echo generated by a 2 meters distant passive tag 45 . Furthermore, while our preliminary measurements already showed that the built SubHT enables longer communication ranges, up to 7 meters, even longer ranges are expected in the future through further technological and design developments. For instance, as we theoretically discussed in ${ }^{27}$, the minimum parametric power threshold exhibited by any varactor-based parametric system is ultimately set by the load and source characteristic impedances that, for SubHTs, represent the input impedances of the adopted antennas. Hence, through the future use of custom low-impedance $(<10 \Omega)$ antenna designs, we anticipate to reduce this power threshold by more than 200 times, thereby enabling much longer communication ranges and higher sensitivities, which will be exclusively limited by the receiver's power sensitivity and by the noise floor of the interrogating nodes.

\section{Conclusions}

We have presented a novel class of chip-less and battery-less tagbased WSNs, named as subharmonic tags (SubHTs). We have showed that SubHTs are inherently able to surpass all the perfor- 


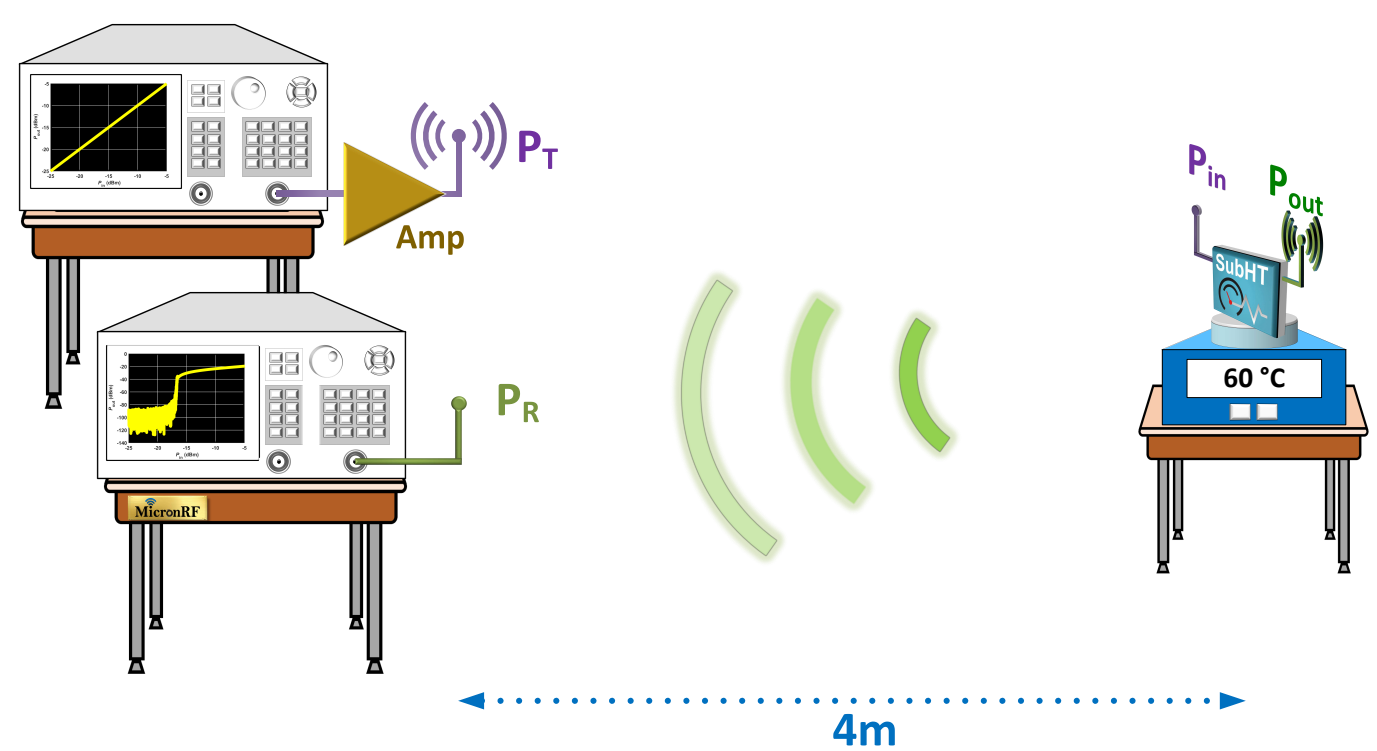

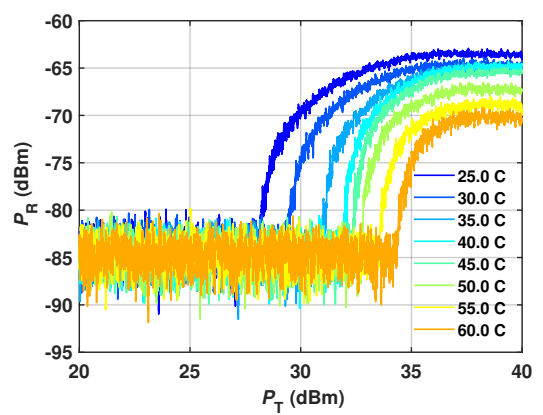

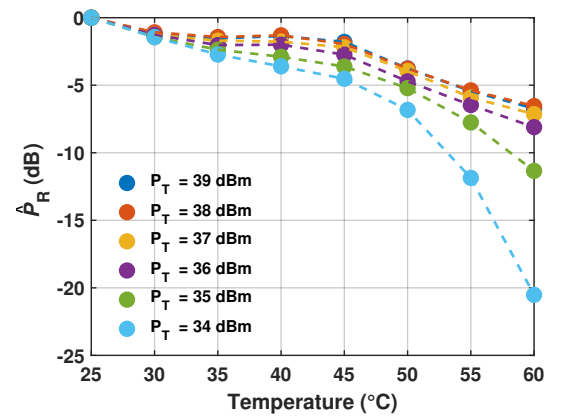

d

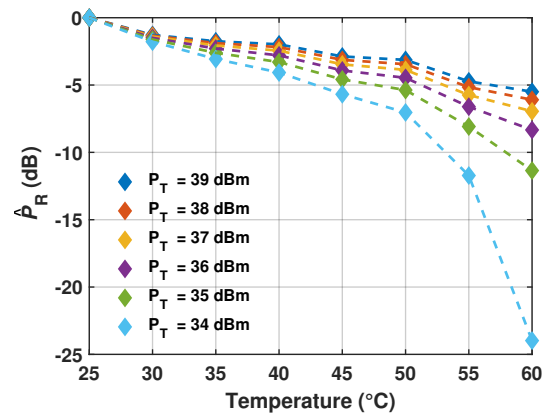

Fig. 6 I Wireless characterization of the built SubHT used as a WSN. a, Overview of the wireless set-up used to characterize the SubHT as a WSN, sensing the local temperature at 4 meters away from two network analyzers placed in the RF test and characterization facility of our group (the MicronRF Laboratory) and together emulating a complementary interrogating node. More details regarding this set-up are provided in the Supplementary Material (Supplementary Fig. 3). b, Measured $P_{R} v s . P_{T}$ trends extracted for the explored temperatures. c,d, Measured (c) and simulated (d) $\hat{P}_{R} v s$. $T$ trends extracted from the wireless characterization of the built SubHT.

mance limitations of the existing harmonic tags. Also, they enable record-high sensitivities and dynamic ranges while being exclusively formed by off-the-shelf components assembled on printed substrates. These unprecedented characteristics were experimentally verified in a standard laboratory setting. In order to do so, we built the first Ultra-High-Frequency SubHT prototype designed to continuously monitor the temperature remotely from an interrogating node. The unique dynamics leveraged by the reported system and discussed for the first time in this article allowed to achieve large, electronically and passively boosted temperature sensitivity and dynamic range, up to $6.2 \mathrm{~dB} /{ }^{\circ} \mathrm{C}$ and $48 \mathrm{~dB}$. These values are respectively 37 and 35,000 times higher than what is possible when the commercial thermistor, selected as the SubHT temperature-sensitive component, is independently used as a temperature sensor for operation within the same explored temperature range. Also, due to its large sensitivity, a minimum temperature resolution of $0.002^{\circ} \mathrm{C}$ was found. The maximum sensitivity achieved by the SubHT highly exceeds the ones attained by state-of-the-art counterparts relying on advanced on-chip manufacturing or on large optical components and systems.

\section{References}

1. Boutry, C. M. et al. Biodegradable and flexible arterial-pulse sensor for the wireless monitoring of blood flow. Nat. biomedical engineering $\mathbf{3}$, 47-57 (2019)

2. Zaky, Z. A. et al. Refractive index gas sensor based on the tamm state in a one-dimensional photonic crystal: Theoretical optimisation. Sci. Reports 10 (2020).

3. Lee, C. et al. Design and application of internet of things-based warehouse management system for smart logistics. Int. J. Prod. Res. 56 , 2753-2768 (2018).

4. Tian, X. et al. Wireless body sensor networks based on metamaterial textiles. Nat. Electron. 2, 243-251 (2019).

5. Jin, J. et al. Evaluation of microclimatic detection by a wireless sensor network in forest ecosystems. Sci. reports 8, 1-9 (2018).

6. Niu, S. et al. A wireless body area sensor network based on stretchable passive tags. Nat. Electron. 2, 361-368 (2019).

7. Karmakar, N., Amin, E. \& Saha, J. Chipless RFID Sensors (John Wiley \& Sons, 2016).

8. Noel, A. B. et al. Structural health monitoring using wireless sensor networks: A comprehensive survey. IEEE Commun. Surv. Tutorials 19, 1403-1423 (2017). 
9. Muhammad, K., Ahmad, J. \& Baik, S. W. Early fire detection using convolutional neural networks during surveillance for effective disaster management. Neurocomputing 288, 30-42 (2018).

10. Molina-Pico, A. et al. Forest monitoring and wildland early fire detection by a hierarchical wireless sensor network. J. Sensors 2016, 1-8 (2016).

11. Jawad, H. M. et al. Energy-efficient wireless sensor networks for precision agriculture: A review. Sensors (Basel, Switzerland) 17 (2017).

12. Qian, Z. et al. Zero-power infrared digitizers based on plasmonically enhanced micromechanical photoswitches. Nat. nanotechnology 12, 969-973 (2017)

13. Mercier, S. et al. Time-temperature management along the food cold chain: A review of recent developments. Compr. Rev. Food Sci. Food Saf. 16, 647-667 (2017).

14. Karray, F. et al. A comprehensive survey on wireless sensor node hardware platforms. Comput. Networks 144, 89-110 (2018).

15. Huang, Q.-A., Dong, L. \& Wang, L.-F. Lc passive wireless sensors toward a wireless sensing platform: Status, prospects, and challenges. $J$. Microelectromechanical Syst. 25, 822-841 (2016).

16. Qian, Z. et al. High figure-of-merit nems thermal detectors based on 50-nm thick aln nano-plate resonators. Appl. Phys. Lett. 115, 261102 (2019).

17. Khan, S. et al. Ultra-low-power chemical sensor node. Proc. GOMAC Tech (2018).

18. Calisgan, S. D. et al. Zero-power chemical sensor based on a poly$\mathrm{mer} / \mathrm{metal}$ micromechanical switch. In Proc. IEEE SENSORS, 1-4 (2019).

19. Nintanavongsa, P. et al. Design optimization and implementation for $\mathrm{rf}$ energy harvesting circuits. IEEE J. on emerging selected topics circuits systems 2, 24-33 (2012).

20. Cansiz, M., Altinel, D. \& Kurt, G. K. Efficiency in rf energy harvesting systems: A comprehensive review. Energy 174, 292-309 (2019).

21. Blampain, E. et al. AlN/sapphire: Promising structure for high temperature and high frequency SAW devices. IEEE Sensors J. 13, 4607-4612 (2013).

22. Kumar, D. et al. Harmonic rfid communication using conventional uhf system. IEEE J. Radio Freq. Identif. 3, 227-235 (2019).

23. Lazaro, A., Villarino, R. \& Girbau, D. A passive harmonic tag for humidity sensing. Int. J. Antennas Propag. (2014).

24. Cassella, C. et al. Parametric filtering surpasses resonator noise in aln contour-mode oscillators. In Proc. IEEE 27th Int. Conf. Micro Electro Mechanical Systems (MEMS), 1269-1272 (2014).

25. Cassella, C. \& Piazza, G. Low phase-noise autonomous parametric oscillator based on a $226.7 \mathrm{MHz}$ AlN contour-mode resonator. Freq. Control. IEEE Transactions on Ultrason. Ferroelectr. 62, 617-624 (2015).

26. Cassella, C. et al. Phase noise suppression through parametric filtering. Appl. Phys. Lett. 110, 063503 (2017).

27. Hussein, H. M. E. et al. Systematic synthesis and design of ultralow threshold 2:1 parametric frequency dividers. IEEE Transactions on Microw. Theory Tech. 1-1 (2020).

28. Melville, R. C. \& Suárez, A. Experimental investigation of bifurcation behavior in nonlinear microwave circuits. IEEE Transactions on Microw. Theory Tech. 65, 1545-1559 (2017).

29. Ramirez, J. C. et al. Polymer based trimodal interferometric sensor. In Proc. SBFoton Int. Optics and Photonics Conf. (SBFoton IOPC), 1-4 (2019).

30. Feng, M. et al. A miniature inline fiber temperature sensor based on a silica capillary partially filled with ethanol. Optik 193, 162993 (2019).

31. Chiang, C.-C., Wu, C.-W. \& Lin, K.-X. Fabrication of electroformed long-period fiber grating by mems process for temperature sensing application. Jpn. J. Appl. Phys. 55, 06GP09 (2016).

32. Wang, F. et al. Comparative study on a core-offset fiber temperature sensor between the faraday rotation mirror structure and the double coupling structure. Opt. Commun. 367, 286-291 (2016).

33. Wang, S. et al. Highly sensitive temperature sensor based on gain competition mechanism using graphene coated microfiber. IEEE Photonics J. 10, 1-8 (2018).

34. Yuan, W. et al. Highly sensitive temperature and humidity sensor based on carbon nanotube-assisted mismatched single-mode fiber structure. Micromachines 10, 521 (2019).

35. Yin, B. et al. High-sensitivity refractive index and temperature sensor based on cascaded dual-wavelength fiber laser and snhns interferometer. Opt. express 27, 252-264 (2019).
36. Sun, X. et al. Graphene coated microfiber for temperature sensor. In Fiber-Based Technologies and Applications, FF4B-3 (Optical Society of America, 2014).

37. Sun, Q. et al. Graphene-assisted microfiber for optical-power-based temperature sensor. IEEE Photonics Technol. Lett. 28, 383-386 (2016)

38. Hernández-Romano, I. et al. Optical fiber temperature sensor based on a microcavity with polymer overlay. Opt. express 24, 5654-5661 (2016).

39. Müller, A. et al. Gan/si based single saw resonator temperature sensor operating in the ghz frequency range. Sensors Actuators, A: Phys. 209, 115-123 (2014)

40. Bruckner, G. \& Bardong, J. Wireless readout of multiple saw temperature sensors. Sensors (Basel, Switzerland) 19 (2019).

41. Tian, X.-G. et al. High-resolution, high-linearity temperature sensor using surface acoustic wave device based on linbo3/sio2/si substrate. AIP Adv. 6, 095317 (2016).

42. Lo, Y. \& Chiu, Y. A high-accuracy, high-resolution, and low-cost alldigital temperature sensor using a voltage compensation ring oscillator. IEEE Sensors J. 16, 43-52 (2016).

43. Szajda, K. S., Sodini, C. G. \& Bowman, H. F. A low noise, high resolution silicon temperature sensor. IEEE J. Solid-State Circuits 31, 1308-1313 (1996).

44. Wang, H. \& Mercier, P. P. Near-zero-power temperature sensing via tunneling currents through complementary metal-oxide-semiconductor transistors. Sci. reports 7, 1-7 (2017).

45. Henry, D., Aubert, H. \& Pons, P. 3D scanning and sensing technique for the detection and remote reading of a passive temperature sensor. In Proc. IEEE MTT-S Int. Microwave Symp. (IMS), 1-4 (2016).

\section{Acknowledgements}

This work has been funded by the National Science Foundation (NSF) under the awarded grant 1854573.

\section{Author contributions}

C.C. conceived the idea and developed the research plan; H.H. designed the device; C.C., H.H., M.R., M.O. contributed to the design of the experiments; H.H. performed the experiments; H.H. and C.C. analyzed the data; H.H., M.R., M.O., C.C. contributed to the preparation and review of the manuscript.

Competing interests

The authors declare no competing interests. 
Figures

a

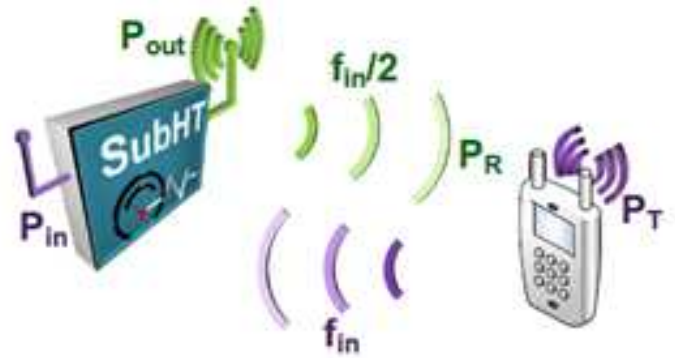

b

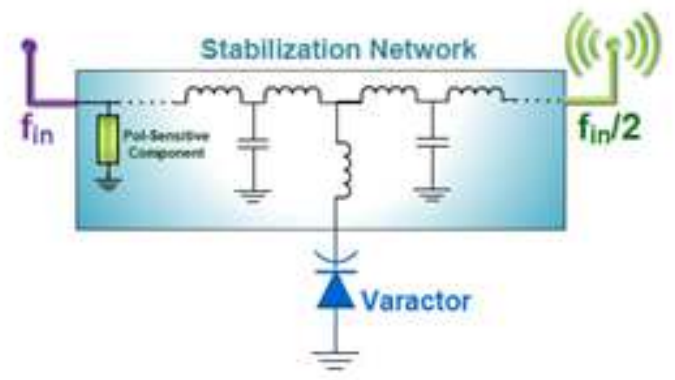

d

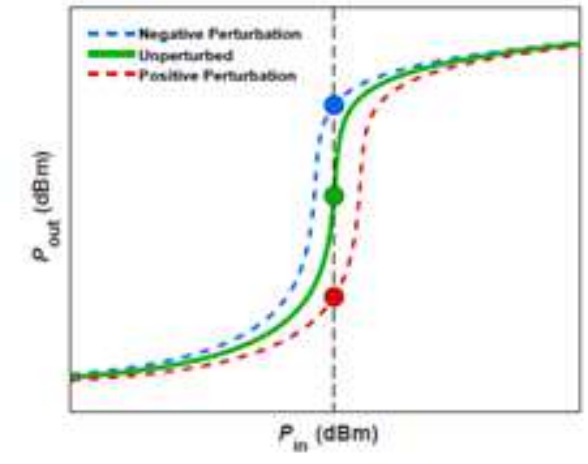

Figure 1

A Sub-Harmonic Tag (SubHT) and its unique operational features 


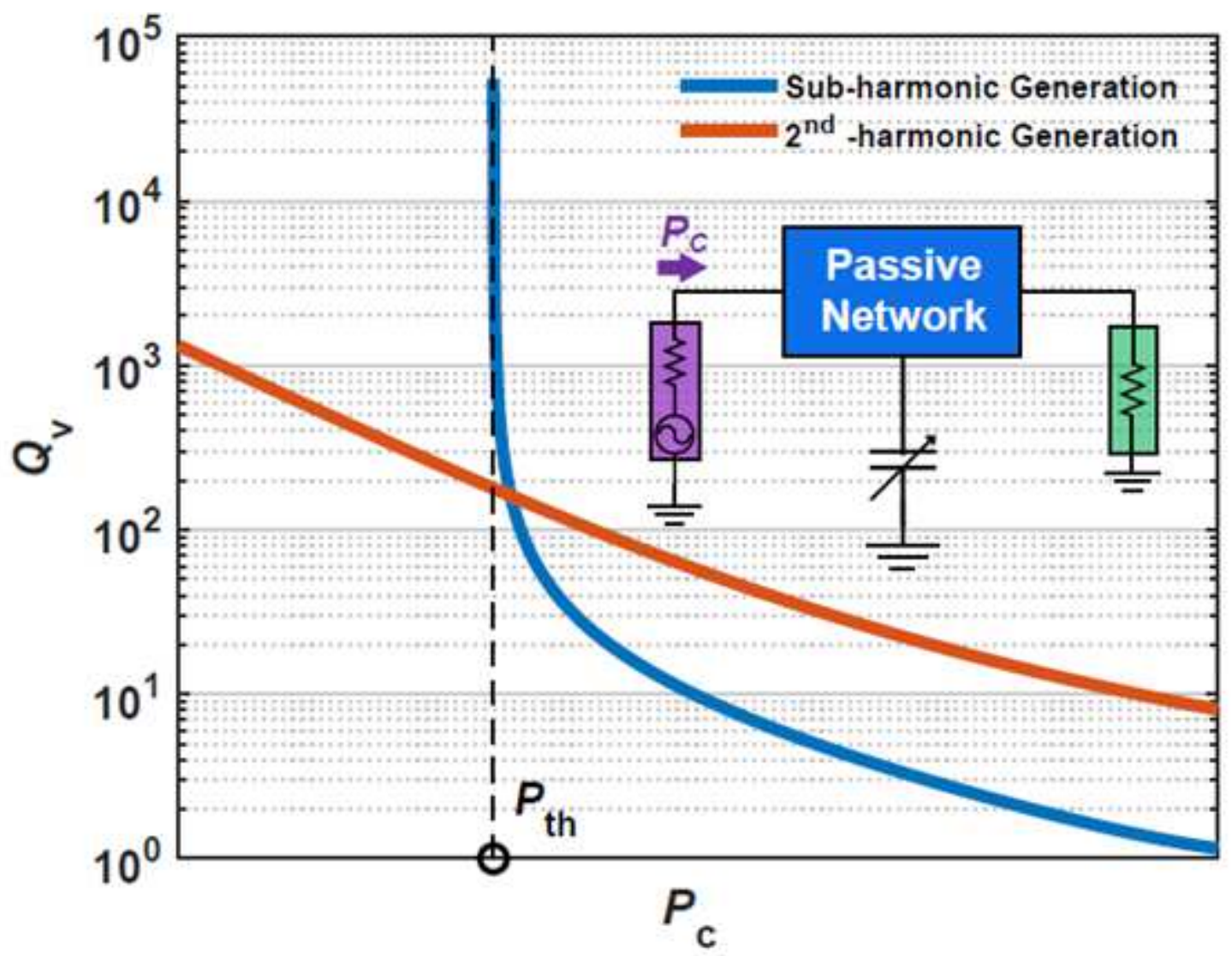

Figure 2

The power dependent quality factor exhibited by an ideal largely modulated reactance used for frequency division or frequency doubling. 


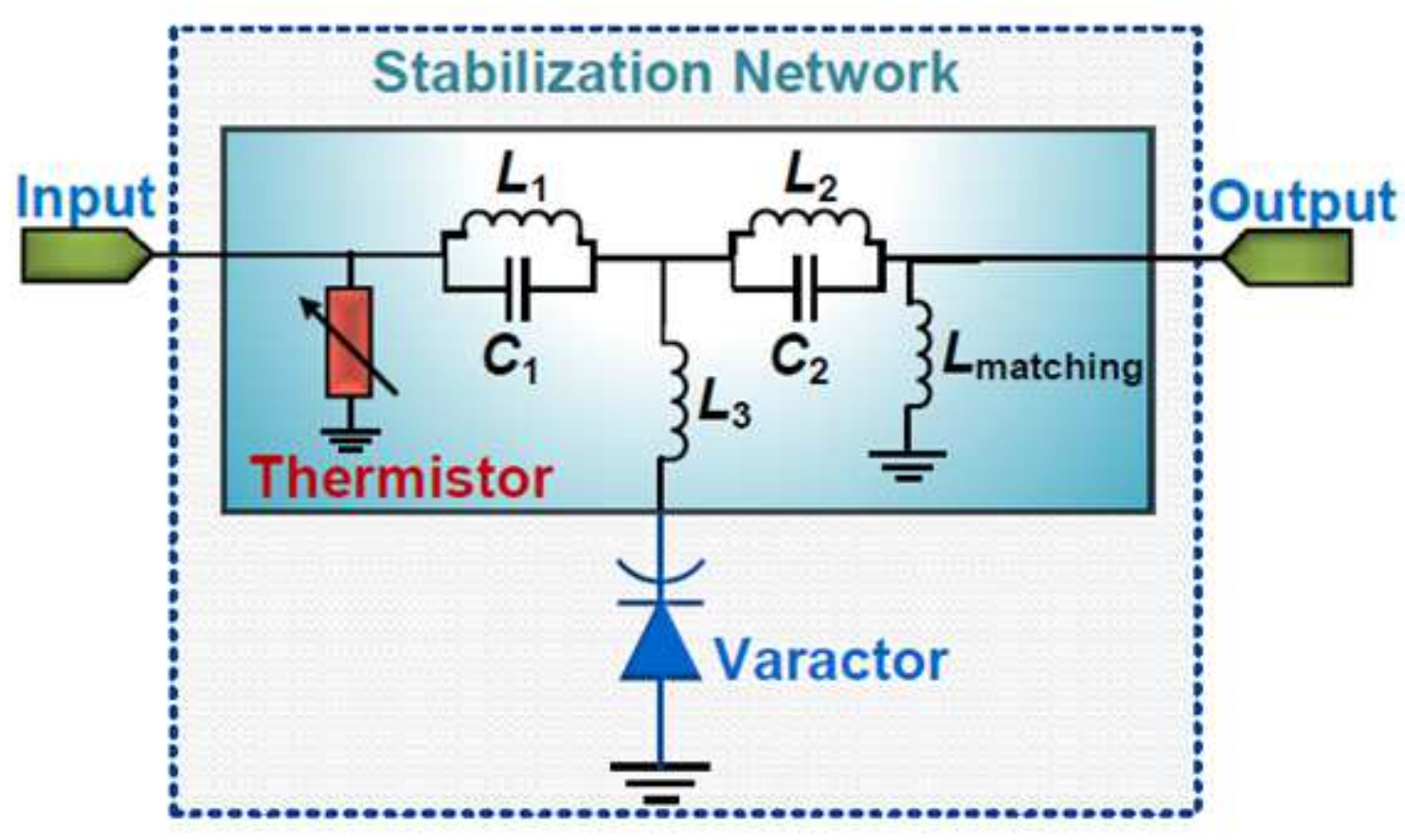

Figure 3

Circuit schematic of the realized SubHT for temperature sensing.

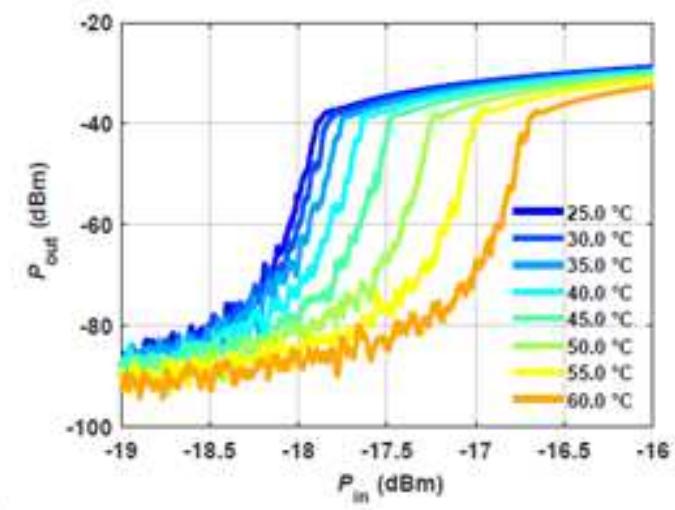

$c$

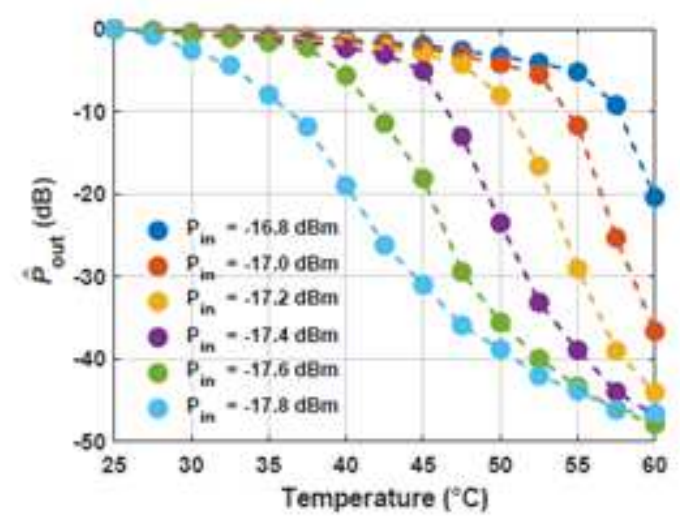

b

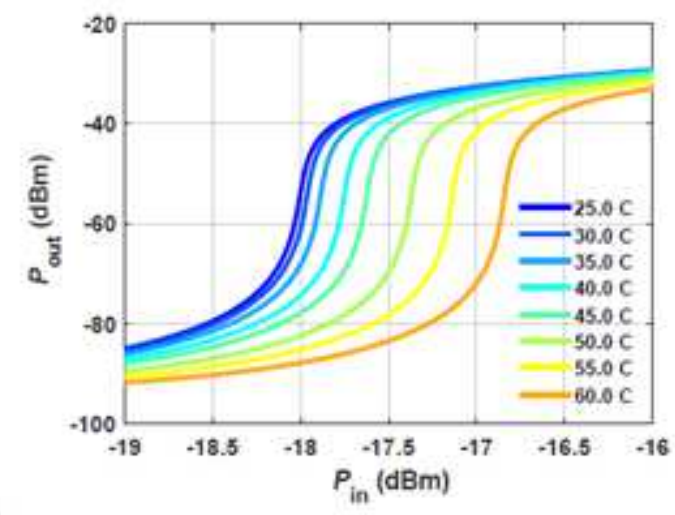

d

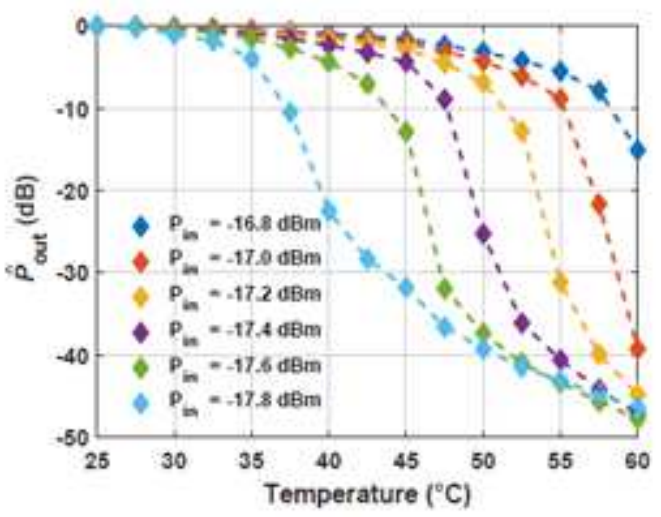


Figure 4

Evaluation of the sensing capabilities of the fabricated SubHT

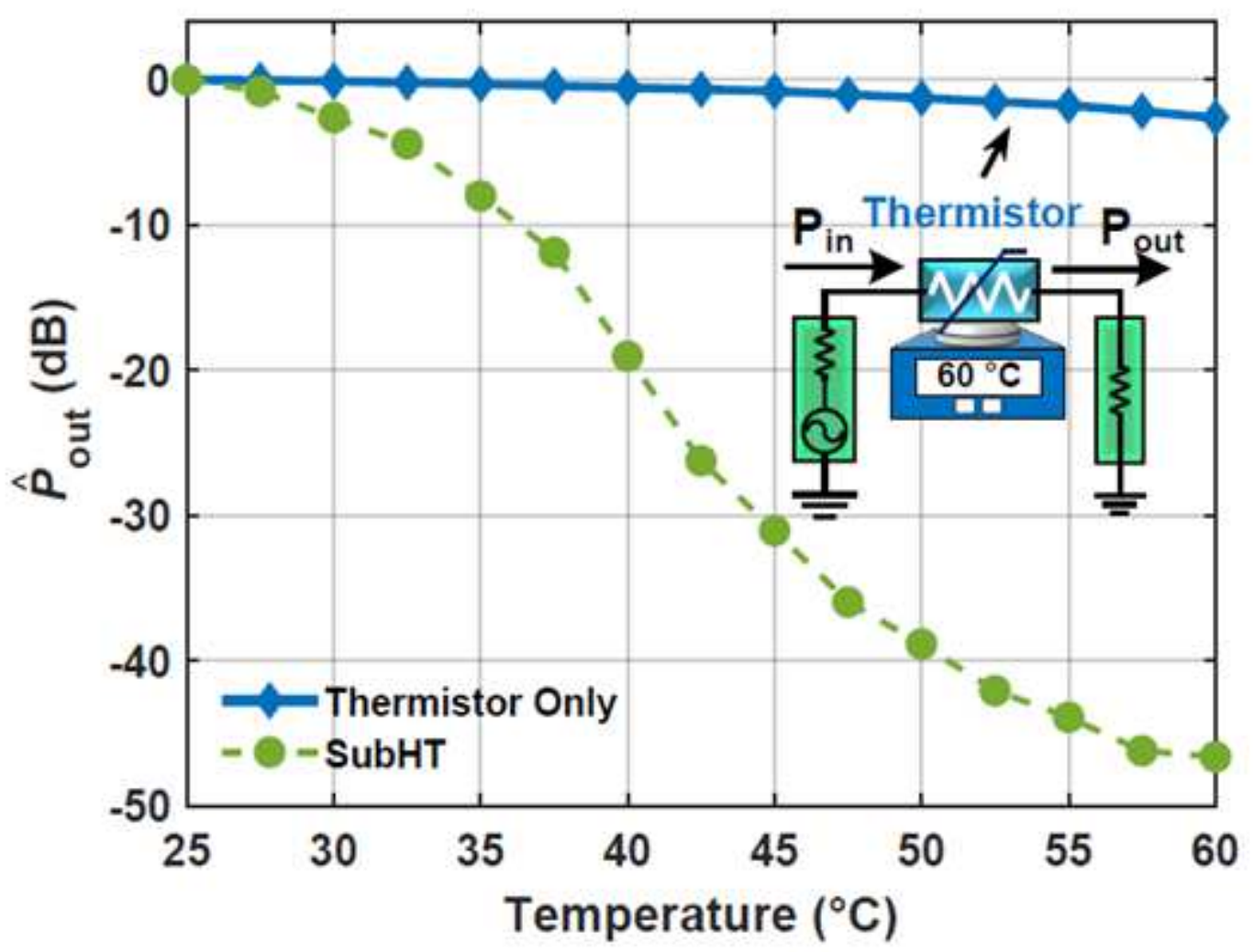

Figure 5

Surpassing the limits in the achievable sensitivity. 
b

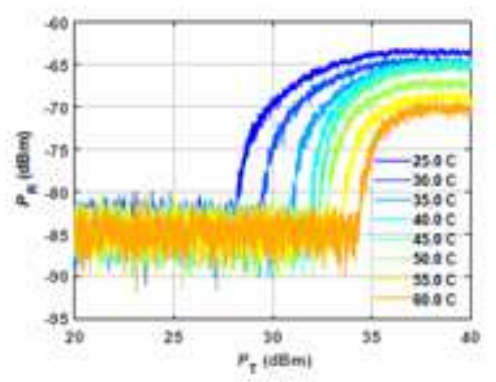

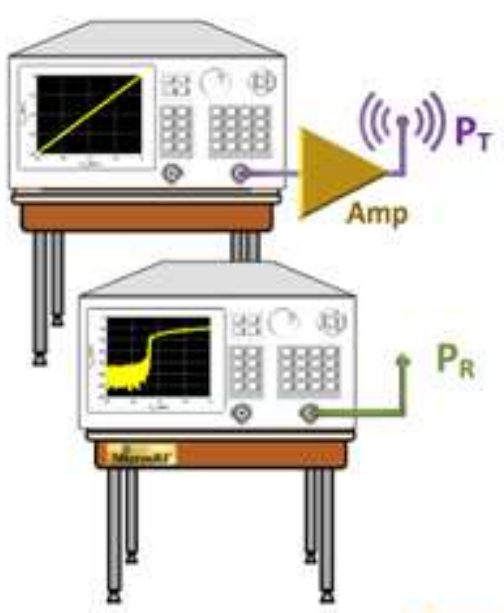

c

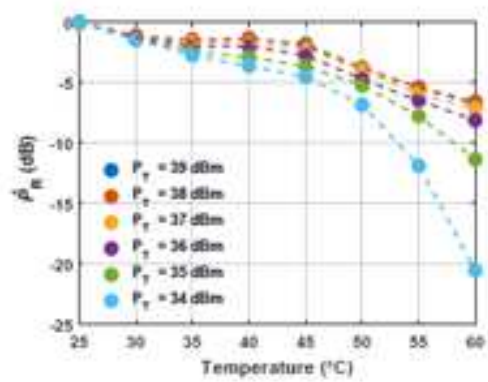

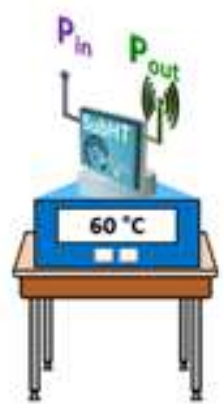

$4 \mathrm{~m}$

d

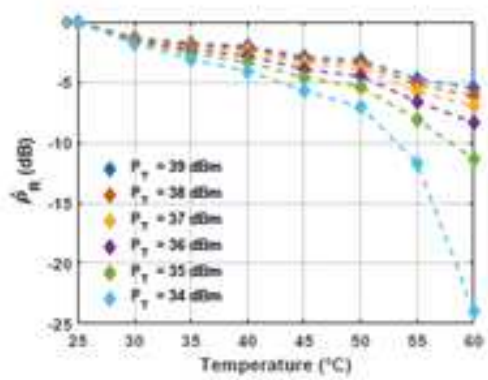

Figure 6

Wireless characterization of the built SubHT used as a WSN.

\section{Supplementary Files}

This is a list of supplementary files associated with this preprint. Click to download.

- Supplementarylnformation.pdf 